\title{
Spectroscopic and photometric observations of the short-period RS CVn star RT And
}

\author{
D. P. Kjurkchieva ${ }^{1}$, D. V. Marchev ${ }^{1}$, and W. Ogloza ${ }^{2}$ \\ 1 Department of Physics, Shoumen University, 9700 Shoumen, Bulgaria \\ and Isaac Newton Institute of Chile, Bulgarian Branch \\ e-mail: d.kyurkchieva@shu-bg.net, d.marchev@shu-bg.net* \\ 2 Mt. Suhora Observatory, Cracow Pedagogical University, 30-084 Cracow \\ and N. Copernicus Astronomical Center, Bartycka 18, 00-716 Warsaw, Poland \\ Received 13 June 2001 / Accepted 23 July 2001
}

\begin{abstract}
Spectroscopic observations in the range 6500-6700 $\AA$ and BVRI photometry of the eclipsing shortperiod RS CVn-star RT And are presented. We determined $K_{1}=130 \mathrm{~km} \mathrm{~s}^{-1}$ and $K_{2}=175.8 \mathrm{~km} \mathrm{~s}^{-1}$ by measurement of the double profiles of the lines $\mathrm{H} \alpha$ and FeI 6678, and obtained the mass ratio $q=0.74$ and masses of the star components $M_{1}=1.23 M_{\odot}$ and $M_{2}=0.91 M_{\odot}$. It was established that the emission activity of RT And is associated with its secondary component. The relative contribution of the secondary star is stronger in the FeI 6678 line than in $\mathrm{H} \alpha$. A strong emission feature between the two profiles of FeI 6678 at phase 0.73 was detected that is probably due to the appearance of extended emitting structure. The multicolor light curve of RT And is fitted by two cool spots on the primary star with equal sizes $21^{\circ}$ and temperatures $4980 \mathrm{~K}$ and located at middle latitudes and longitudes symmetrical to the line connecting the star centers. Both spectral and photometric data lead to the conclusion that the secondary star of RT And is oversized for its mass.
\end{abstract}

Key words. stars: activity - binaries: eclipsing - binaries: spectroscopic - stars: chromospheres stars: individual: RT And - stars: starspots

\section{Introduction}

The eclipsing binary star RT And has been observed since the beginning of 20 th century, however it only become the subject of extensive photometric observations after 1970. RT And is currently considered to be a member of the short-period RS CVn group. Hall (1976) defined the RS CVn stars as noncontact binaries in which the primary (hotter) star had spectral type F-G V-IV, and at least one component showed CaII $\mathrm{H}$ and $\mathrm{K}$ emission. Budding \& Zeilik (1987) concluded that both components of the short-period RS CVn's are main-sequence stars, in contrast to the main group which contains active subgiant components.

The strong photospheric, chromospheric, transition region, and coronal activity in RS CVn stars are generally attributed to the deep convection zone and the fast rotation that drives the dynamo mechanism. Enhanced emission cores in the CaII $\mathrm{H}$ and $\mathrm{K}$ lines and $\mathrm{H} \alpha$ line are the primary optical indicators of the chromospheric activity (Barden 1985; Fernandez-Figueroa et al. 1994).

\footnotetext{
Send offprint requests to: Waldemar Ogloza,

e-mail: ogloza@ap.krakow.pl

* Research supported in part by grants from Foundation Queen Jadwiga of the Jagiellonian University, Cracow, Poland.
}

The analysis of the spectra at different orbital phases allow to establish which star is the source of the emission.

The variability of the light curves of RT And in the visual range is exhibited by the variable depths of the eclipses and irregular changes of the brightness around the quadratures. These photometric variations have been successfully interpreted as the effect of large cool starspots on the surface of the primary component. Although it is generally accepted that the starspots are the main source of photometric variability of RT And, the presence of narrow features (depressions) at the beginning and end of the eclipses (Dean 1974; Milano 1981, 1986; Arevalo \& Lazaro 1995) cannot be explained in terms of starspots or flares but might due to transient extinction effects of circumstellar structures. The observed night-to-night light fluctuations (Gordon 1955) probably originate also from extraphotospheric structures.

In the UV range RT And presents emission in the $\mathrm{Mg} \mathrm{H}$ and $\mathrm{K}$ lines, indicating a high degree of chromospheric activity (Budding et al. 1982).

In this paper we report the results of our investigation of the appearances of star activity of the components of RT And on the basis of our spectroscopic and photometric observations. 


\section{Observations}

\subsection{Spectroscopy}

RT And was observed in the spectral range $6500-6700 \AA$ with resolution $0.19 \AA /$ pixel on 3 consecutive nights at the end of November 1999. We used a CCD camera mounted on the Coude spectrograph (grating B\&L632/14.7 ${ }^{\circ}$ ) of the 2-m telescope operated by the National Astronomical Observatory in Rozhen (Bulgaria). The seeing during the observations did not exceed $2 \operatorname{arcsec}(F W H M)$. The exposure time was $20 \mathrm{~min}$. The bias frames and flat-field integrations were obtained at the beginning and end of each night. All stellar integrations were alternated with Fe comparison source exposures. The data were processed in a standard way using the PCIPS (Smirnov et al. 1992) and Rewia (Borkowski 1988) software packages. The ratio $S / N$ was around $120-160$. There are several weaker spectral lines in the range $6500-6700 \AA$ that show doubling out of the eclipses as well as $\mathrm{H} \alpha$. We chose to investigate the line FeI $6678 \AA$ (see discussion about its identification further).

An appropriate Fourier noise filter was applied to the weaker line Fe I 6678 in order to remove the high-frequency noise. This procedure causes some smoothing of the profiles without loss of their main details (Gray 1992).

The spectra were reduced by bias substraction, flatfield division and wave-length calibration. The normalization was made on the whole line-free spectral range.

The data were phased by the ephemeris of Dapergolas et al. (1991)

$\mathrm{JHD}(\mathrm{MinI})=2447803.5094+0.6289294 * E$.

The normalized $\mathrm{H} \alpha$ and $\mathrm{FeI} 6678$ profiles are displayed in Figs. 1 -5 and Figs. 6-10 respectively together with the corresponding orbital phases.

\subsection{Photometry}

Our BVRI photometry of RT And in 1998 was obtained with two-channel photometer mounted on the $60-\mathrm{cm}$ telescope of the Mt. Suhora Observatory. The photometer is equipped with an autoguider (Krzesinski \& Wojcik 1993). We used HD 219038 as a comparison star and HD 218915 as a check star. Calibration of the channels was made every night. The integration times were $10 \mathrm{~s}$ in $B, V, R$ bands and $20 \mathrm{~s}$ in $I$.

Figure 11 presents the complete light curve of RT And. The errors of the individual points do not exceed 0.01 in $B, V, R$ colors and 0.02 in $I$ color (due to the bigger sky level and lower sensitivity of the detector in $I$ band).

Our data have high time resolution and full phase coverage and thus photometric analysis possible.

\section{Results}

\subsection{Analysis of the spectral data}

The measurement of the lines of the short-period RS CVn systems is difficult because the profile arising

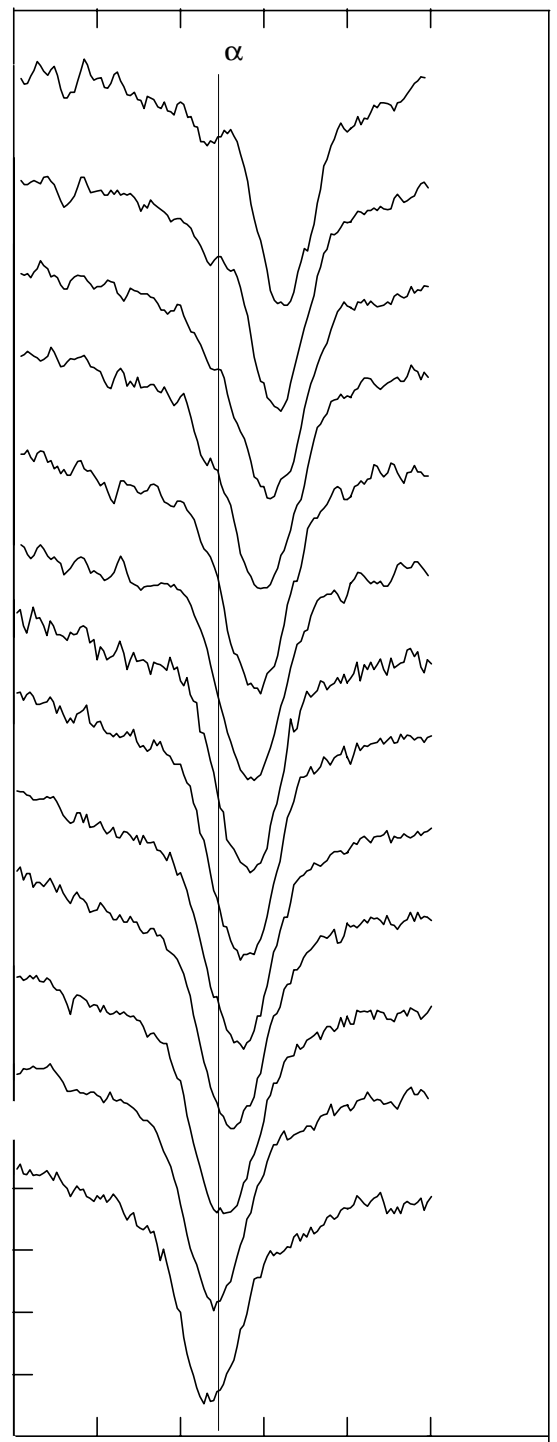

Fig. 1. H $\alpha$ profiles of RT And from Nov. 28.

from each component is broadened, radially velocityshifted and blended with the surrounding metal lines of itself and its companion star, and because of presence of emission features.

The second line of RT And in the range 6500-6700 $\AA$ with well pronounced doubling out of the eclipses is the line $6678 \AA$. In the framework of our spectral resolution we cannot identify it by the wavelength only because there are three lines with almost equal wavelengths belonging to FeI $(6677.99 \AA)$, HeI $(6678.15 \AA)$ and $\mathrm{ZrI}(6678.01 \AA)$. The line FeI 6678 with $\chi=2.69 \mathrm{eV}$ is strong in the solar types spectra. It is generally assumed that this line has to be there in spectra of $\mathrm{F}$ to $\mathrm{K}$ stars, unless these stars are very metal deficient. In contrast, the line Zr I 6678 $(\chi=2.41 \mathrm{eV})$ is not visible in any of the atlas spectra probably because it hides within the stronger Fe line (the normal abundance ratio of Fe to $\mathrm{Zr}$ is 100 000). Similarly, it is well known that stars later than spectral type A do not show any lines of He. The line HeI $6678 \AA$ corresponds 


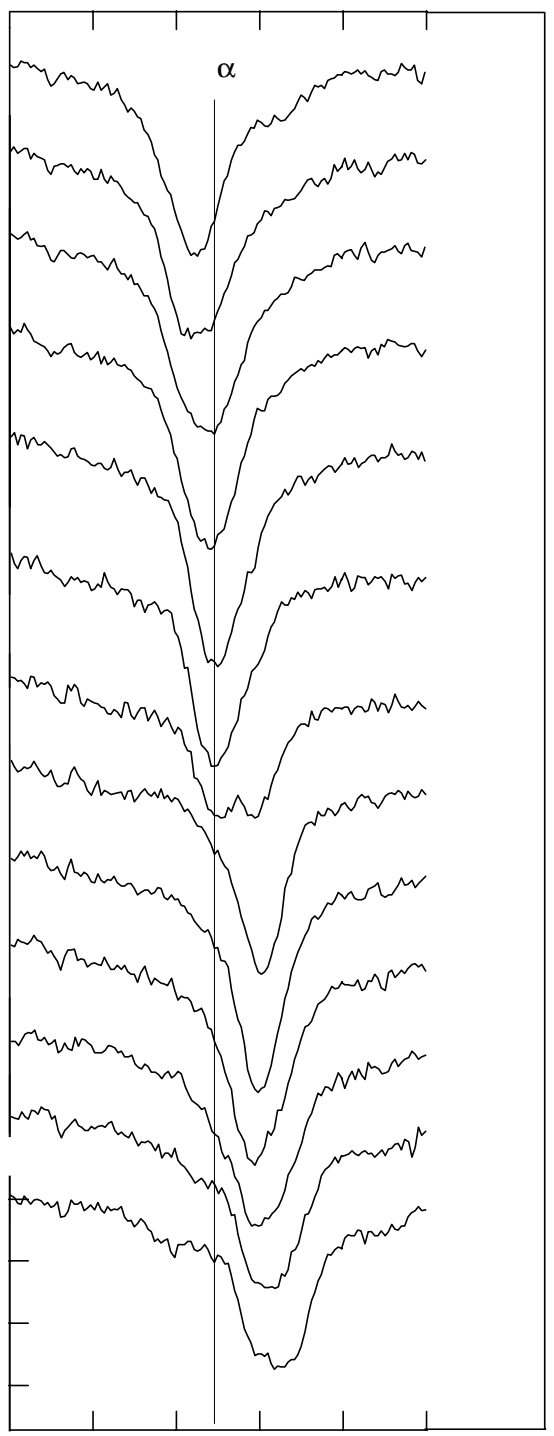

Fig. 2. $\mathrm{H} \alpha$ profiles of RT And from Nov. 29.

to the transition from the state $2 \mathrm{P}$ to the state $3 \mathrm{D}$ of the $\mathrm{HeI}$ atoms. But the probability of $\mathrm{HeI}$ atoms to be at state $2 \mathrm{P}$ is very small because its big excitation energy (around $21 \mathrm{eV})$. So, we assume that the line $6678 \AA$ originates mostly from FeI.

The absorption lines H $\alpha$ and FeI 6678 of RT And show double profiles outside the eclipses (Figs. 1-10). Although the line FeI 6678 is weaker than $\mathrm{H} \alpha$ its doubling is better pronounced because the line $\mathrm{H} \alpha$ has broad wings. The radial velocities were determied by fitting each profile with 6 th-order polynomial and measuring the wavelength position of the midpoint at half height. The radial velocity curves for the $\mathrm{H} \alpha$ and FeI 6678 lines were fitted by sinusoids (Fig. 12). The results of the least squares analysis of the data are: $K_{1}(\mathrm{H} \alpha)=129.54 \pm 0.75 \mathrm{~km} \mathrm{~s}^{-1}$; $K_{2}(\mathrm{H} \alpha)=173.51 \pm 1.46 \mathrm{~km} \mathrm{~s}^{-1} ; \gamma(\mathrm{H} \alpha)=19.65 \pm$ $1.19 \mathrm{~km} \mathrm{~s}^{-1} ; K_{1}(\mathrm{FeI})=130.49 \pm 0.96 \mathrm{~km} \mathrm{~s}^{-1} ; K_{2}(\mathrm{FeI})=$ $177.56 \pm 1.05 \mathrm{~km} \mathrm{~s}^{-1} ; \gamma(\mathrm{FeI})=19.62 \pm 1.08 \mathrm{~km} \mathrm{~s}^{-1}$. The weight-averaged values are correspondingly $K_{1}=$ $130 \pm 0.8 \mathrm{~km} \mathrm{~s}^{-1}$ and $K_{2}=175.8 \pm 1.2 \mathrm{~km} \mathrm{~s}^{-1}$.

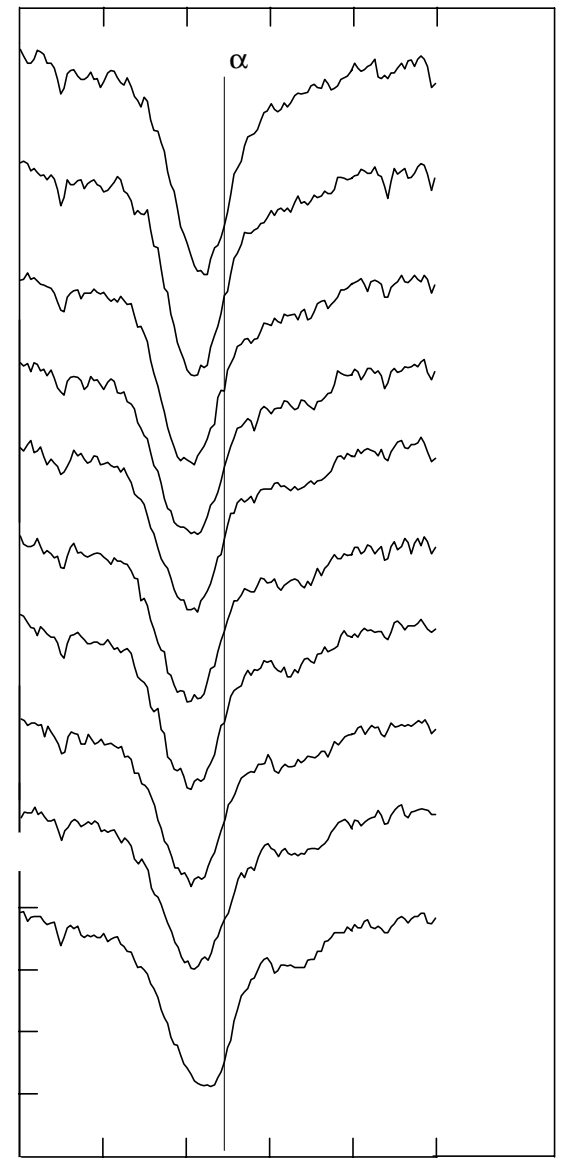

Fig. 3. $\mathrm{H} \alpha$ profiles of RT And from Nov. 29, continuation.

They are slightly smaller than those determined by Popper (1994).

The calculated values of the mass ratio and masses of the star components of RT And from our mean values of $K_{1}$ and $K_{2}$ are: $q=0.74 ; M_{1}=1.23 M_{\odot} ; M_{2}=0.91 M_{\odot}$. The corresponding values of the radii of the star components of RT And on the mass-radius relation for MS stars are $R_{1}=1.26 R_{\odot}$ and $R_{2}=0.92 R_{\odot}$ and respectively their ratio is $k=R_{2} / R_{1}=0.73$. These values are very close both to those obtained by Arevalo \& Lazaro (1995) and those of Popper (1994) (see Table 2 further).

The FWHM of the profiles of the primary and secondary star are on average 4.7 and $3.7 \AA$ for $\mathrm{H} \alpha$ but 3.4 and $3 \AA$ for FeI 6678 .

Because the tidal interactions induce synchronous rotation in the short-period RS CVn systems we can calculate the equatorial velocities of the star components of RT And using the determined star radii ( $V_{\mathrm{eq}}=$ $\left.2 \pi R / P_{\text {rot }}\right)$. The obtained values are: $V_{\text {eq }}^{1}=101 \mathrm{~km} \mathrm{~s}^{-1}$ and $V_{\mathrm{eq}}^{2}=74 \mathrm{~km} \mathrm{~s}^{-1}$.

The rotation broadenings of the spectral lines $\mathrm{H} \alpha$ and FeI 6678 corresponding to these equatorial velocities are 4.42 and $4.5 \AA$ for the primary profile and 3.24 and $3.3 \AA$ for the secondary one (RT And is eclipsing binary and we can assume $\sin i \simeq 1$ ). Because the Doppler broadening dominates the cores of the line profiles we determined 


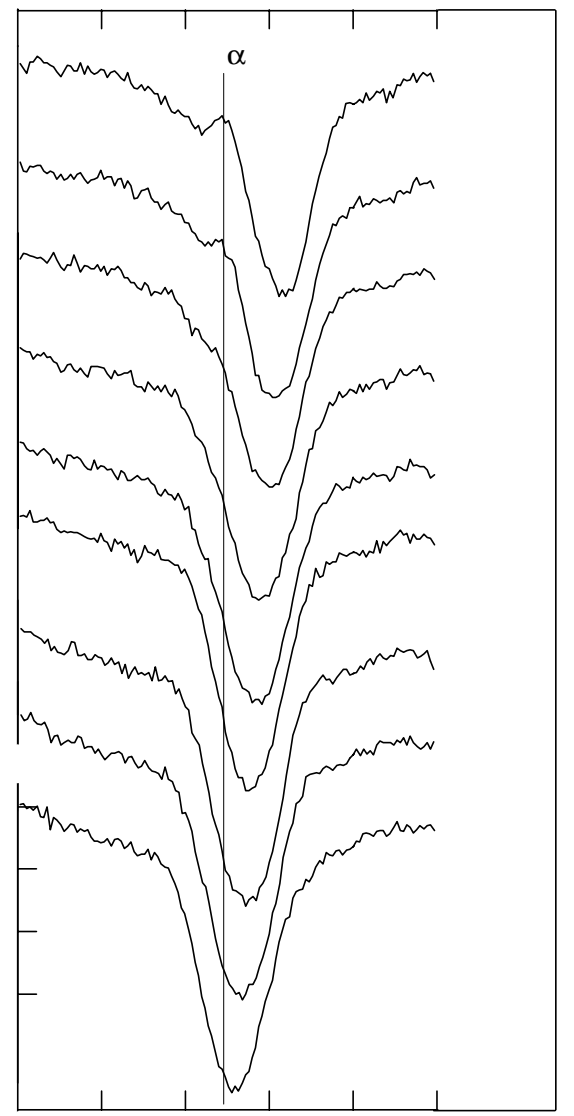

Fig. 4. $\mathrm{H} \alpha$ profiles of RT And from Nov. 30.

the actual rotation broadenings of the two components of the investigated lines by measurement of the widths of the parabolas fitting the central parts of these profiles at the continuum level. The measured values are almost the same for the $\mathrm{H} \alpha$ and FeI 6678 line: around $4.5 \AA$ for the primary profile and $3.8 \AA$ for the secondary one. Therefore, the measured and calculated values for the primary star coincide, while the measured rotational broadenings of the secondary profiles are larger than the calculated ones. The ratio of the star radii of RT And corresponding to the measured rotational broadenings of their profiles is $k=0.84$. This fact might be attributed to the greater value of the radius of the secondary star than that obtained from the mass-radius relation of the MS stars, i.e. this star should be "oversized" for its mass. A similar conclusion was reached earlier by Arevalo \& Lazaro (1995) on the base of an modeling of the light curve of RT And.

For comparison, by measurement of the line widths of RT And Popper (1994) determined a rotational velocity of $100 \mathrm{~km} \mathrm{~s}^{-1}$ for the primary star and $80 \mathrm{~km} \mathrm{~s}^{-1}$ for the secondary component while Huisong \& Xuefu (1987) measured $109 \mathrm{~km} \mathrm{~s}^{-1}$ for the primary star.

The central intensities $R_{\mathrm{c}}=1-I_{\text {line }} / I_{\text {cont }}$. of the investigated double lines changes during the orbital cycle in the ranges $0.25-0.4$ and $0.05-0.07$ of the two profiles of $\mathrm{H} \alpha$ and $0.015-0.02$ and $0.005-0.008$ for FeI.

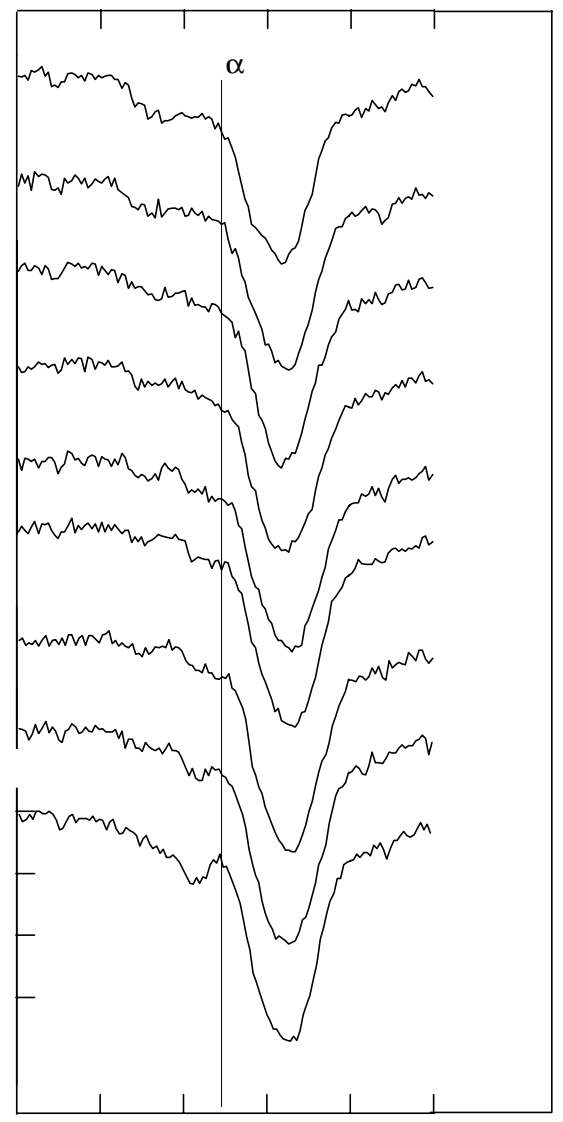

Fig. 5. $\mathrm{H} \alpha$ profiles of RT And from Nov. 30, continuation.

We established the following trends in the phase behavior of $R_{\mathrm{c}}(\mathrm{H} \alpha)$ of the primary star: (a) it is considerably bigger at the phase range around the second quadrature than around the primary one; (b) it is biggest during the secondary eclipse. We do not discuss the phase behavior of the central intensities $R_{\mathrm{c}}$ of the rest profiles because they are affected by emission features.

The chromospheric activity level of a star can be inferred by the presence of emission or filled-in absorption in the line cores. Our spectroscopy of RT And shows a filled-in $\mathrm{H} \alpha$ profile from its primary star only at phases 0.11 and $0.86-0.88$. These phases are just after and before the primary eclipse. Similarly, Popper (1994) found $\mathrm{H} \alpha$ emission from the primary star of RT And only at the phase 0.88. Probably this spectral feature is permanent. The $\mathrm{H} \alpha$ profiles from the secondary star in the phase range 0.68-0.82 have emission cores. Consequently, the weak $\mathrm{H} \alpha$ emission of RT And should be associated rather with the secondary star.

The profiles of the double FeI 6678 line have emission features at the most phases out of the eclipses that are better pronounced on the profiles of the secondary star (similarly to the line $\mathrm{H} \alpha$ ). We noted a trend the emission cores of the profiles of FeI of the secondary star to be stronger around the second quadrature (phase 0.75) than around the primary one, similarly to the $\mathrm{H} \alpha$ line. 


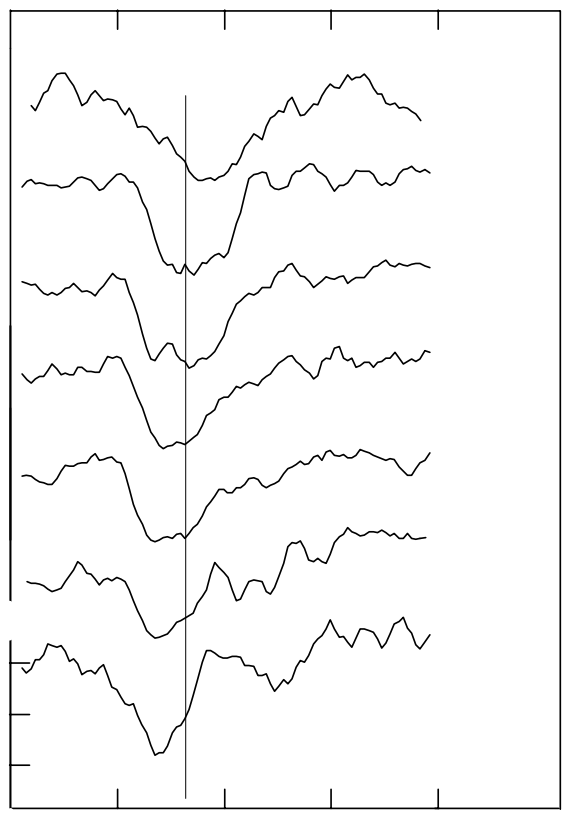

Fig. 6. FeI 6678 profiles of RT And from Nov. 28.

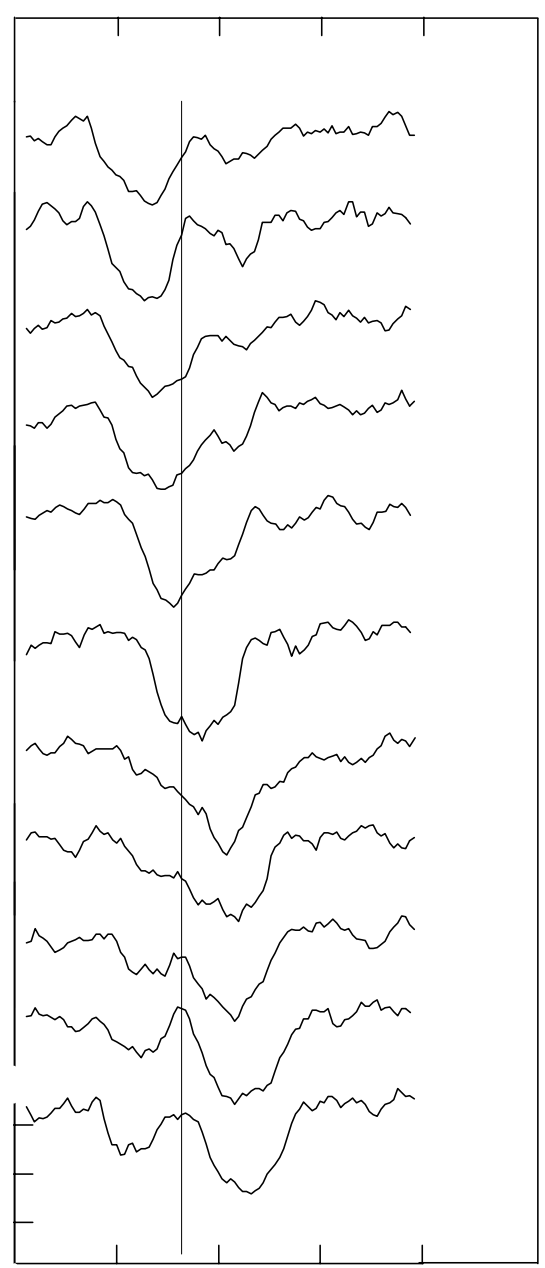

Fig. 7. FeI 6678 profiles of RT And from Nov. 29.

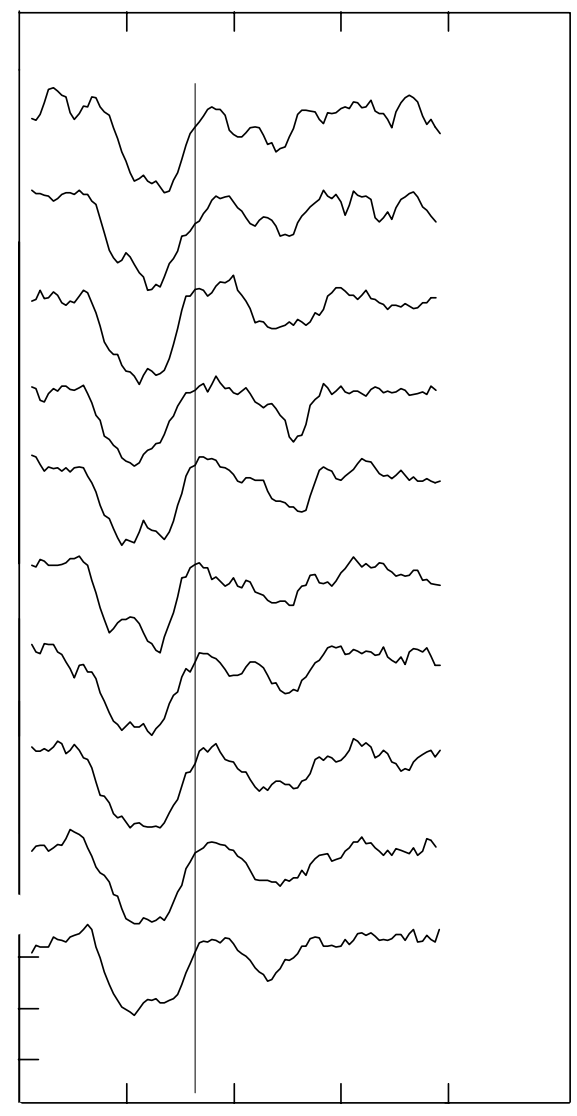

Fig. 8. FeI 6678 profiles of RT And from Nov. 29, continuation.

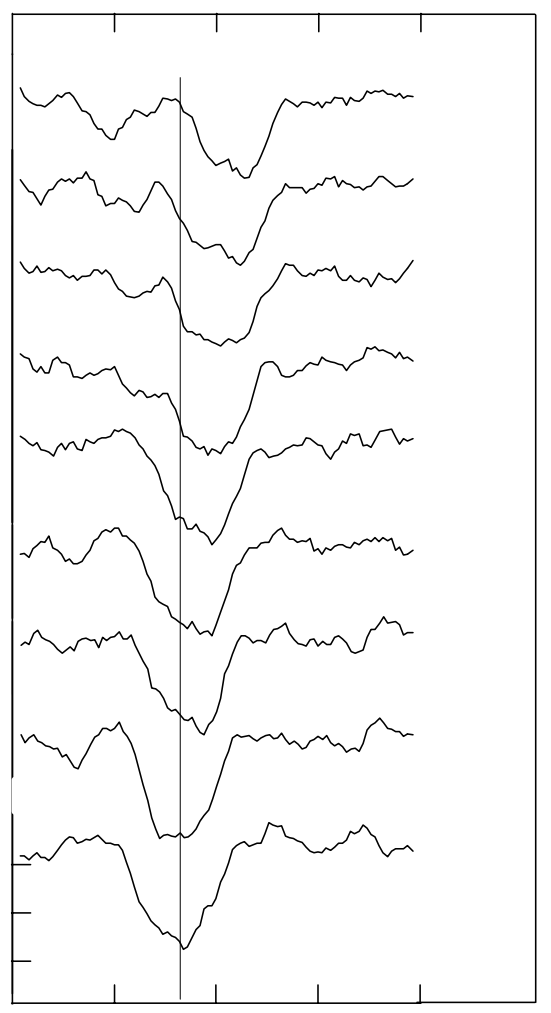

Fig. 9. FeI 6678 profiles of RT And from Nov. 30. 


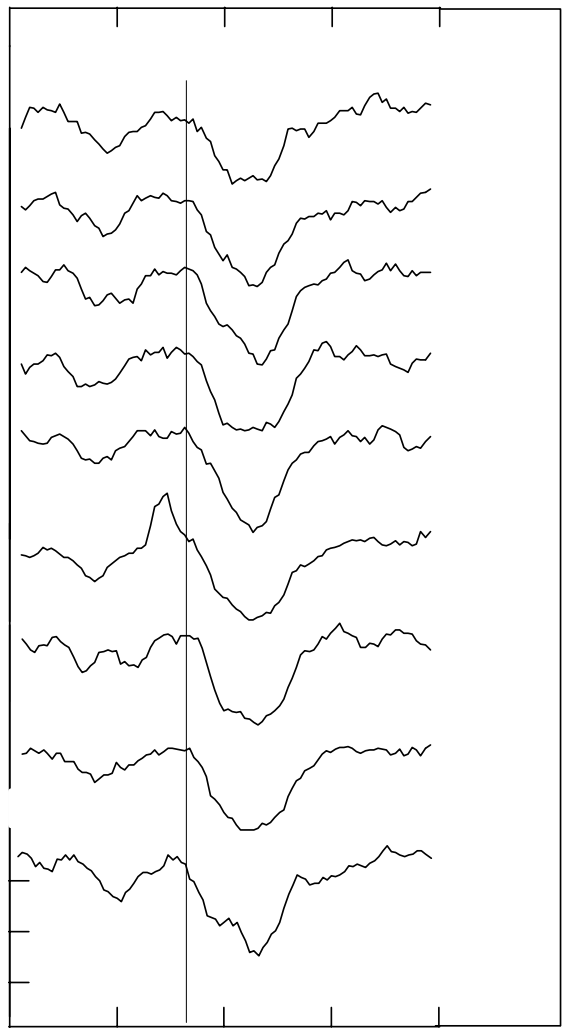

Fig. 10. FeI 6678 profiles of RT And from Nov. 30, continuation.

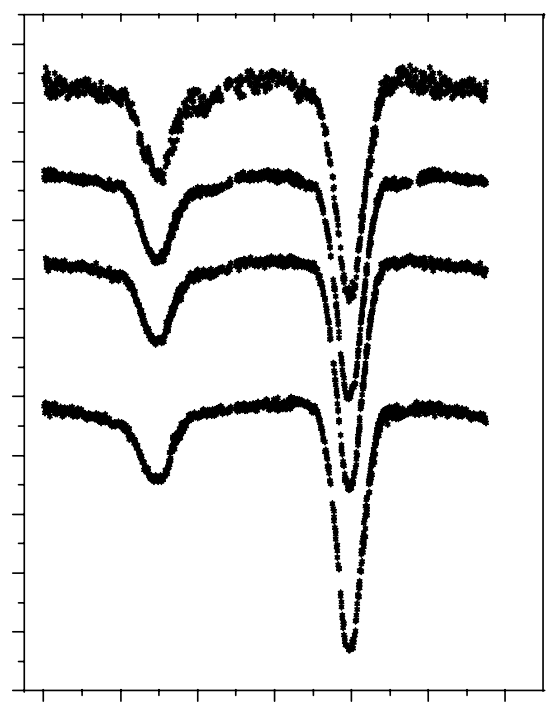

Fig. 11. Multicolor light curve of RT And during 1998.

The measured equivalent widths of the absorption profiles of the primary and secondary star are: $E W_{1}(\mathrm{H} \alpha)=$ $1.12 \AA ; E W_{2}(\mathrm{H} \alpha)=0.19 \AA ; E W_{1}(\mathrm{FeI})=0.114 \AA ;$ $E W_{2}(\mathrm{FeI})=0.033 \AA$. So, the ratio $l$ of the line fluxes from the secondary and primary star is around 0.17 for the line $\mathrm{H} \alpha$ and 0.29 for FeI 6678 .
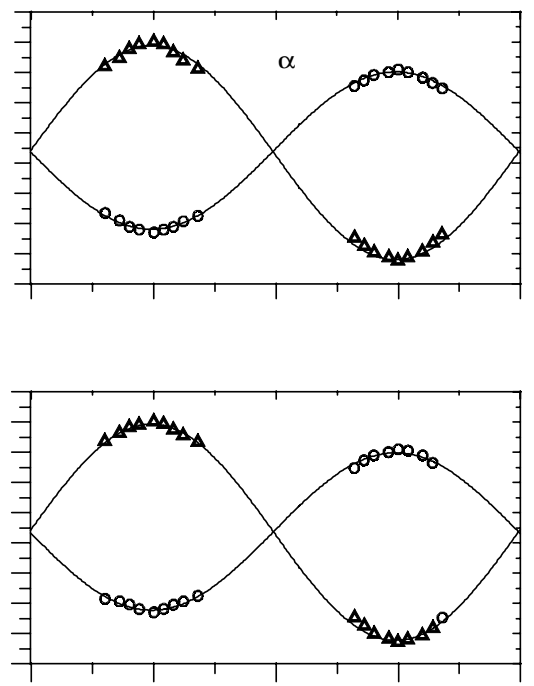

Fig. 12. Radial velocity curves of RT And.

The measured equivalent widths of the strongest emission cores of the profiles of the primary and secondary star during the orbital cycle reach to $0.035 \AA$ and $0.075 \AA$ for $\mathrm{H} \alpha$ and $0.04 \AA$ and $0.012 \AA$ for FeI 6678. So, the maximum ratios of the emission to the absorption flux for the primary and secondary profile are: 0.03 and 0.39 in $\mathrm{H} \alpha$ and 0.35 and 0.36 in FeI 6678.

We detected a strong emission feature between the two profiles of FeI 6678 at phase 0.73 . Probably it is due to transient extended emitting structure (flare or prominence) because it does not repeat after half a period. Taking into account the short existence of this feature we attributed it rather to a flare. Similarly, a third emission component but in the line $\mathrm{H} \alpha$ of the short-period RS CVn stars XY UMa and WY Cnc was found at some orbital phases (Arevalo \& Lazaro 1999) as well as in the spectra of UV Psc (Barden 1985; Hall \& Ramsey 1992).

The relative low ratio $S / N$ of our spectral data does not satisfy the strong requirements of the Doppler imaging technique for surface mapping of the star components on the base of spectral data. For this aim we used the photometric data.

\subsection{Analysis of the photometric data}

The times of minima of our light curve of RT And were determined by the method of Kwee \& Woerden (1956). They are:

$$
\begin{aligned}
& \text { MinI }=2451032.43135 \pm 0.00005 \\
& \text { MinII }=2451036.5189 \pm 0.0005
\end{aligned}
$$

It should be noted that the times of the minima in the different colors coincide to within the errors. They are separated by half an orbital period, which means that the orbit of RT And is a circle. The radial velocity curves (Fig. 12) lead to the same conclusion. 
Fourier analysis of our photometric data does not show any light variabilities with periods less than the orbital period.

The two minima of our light curve are almost symmetrical except for increasing branch of the secondary minimum in $B$, which is slightly steeper than the decreasing one. Dapergolas et al. $(1991,1994)$ note also that the asymmetry of the secondary minimum gets larger towards shorter wavelengths.

Figure 11 shows that the brightness of RT And during 1998 decreases almost linearly after the primary minimum and increases almost linearly after the secondary minimum. This behavior of our light curve out of the eclipses is just opposite to that in 1992 (Dapergolas et al. 1994) when the brightness increased almost linearly after the primary minimum and decreased almost linearly after the secondary minimum.

The depths of the primary minimum of our light curve are $0.82,0.77,0.75$ and $0.7 \mathrm{mag}$ in the $B, V, R$ and $I$ bands respectively. The values are slightly smaller than those of Heckert (1995) and Heckert et al. (1996). The depths of the secondary minimum of our observational light curve are $0.25,0.27,0.3$ and $0.33 \mathrm{mag}$ in the $B, V, R$ and $I$ bands respectively. They are slightly different than those observed previously (Zeilik et al. 1988; Heckert 1995; Heckert et al. 1996).

There are two small depressions of the brightness in the phase ranges $0.1-0.2$ and $0.6-0.7$ that are deeper at the longer wavelengths. Arevalo \& Lazaro (1995) point out that similar photometric depressions at the end of the eclipses are frequently observed in many detached and semidetached binaries. We found similar features in the light curves of other two short-period RS CVn stars: XY UMa (Kjurkchieva et al. 2000a) and SV Cam (Kjurkchieva et al. 2000b).

The energy distributions of the optical emission of RT And at the primary minimum MinI, the secondary minimum MinII, the quadrature Q1 (after MinI) and the quadrature Q2 (after MinII) are given in Fig. 13. They may be approximated roughly as $F_{\lambda}=$ const. $\lambda^{k}$ where the $k$ values are $-1.06,-1.25,-1.33$ and -1.18 for MinI, Q1, MinII and Q2, respectively. Consequently, the system becomes redder at the primary minimum and bluer at the secondary minimum, in correspondence with the spectral types of its components.

The measured color index $B-V=0.47$ of the primary star is almost the same as that obtained by Dapergolas et al. (1991). It is bluer than the expected value 0.540.57 for the corresponding spectral type. But this result is not surprising: Arevalo \& Lazaro (1995) noted that the active stars show blue excess but the reason for this effect is unknown.

In order to model our multicolor light curve of RT And we used the packages of Wilson $(1979,1983)$ and Bradstreet (1993) for a light curve synthesis.

The modeling procedure was made in three consecutive steps because too many parameters of the system were unknown.

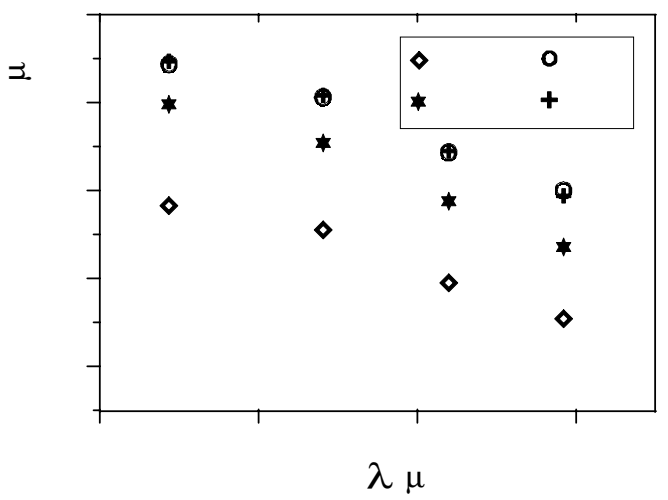

Fig. 13. Energy distribution of RT And at the light minima and quadratures.

We only fixed in advance the value $q=0.74$, obtained from the analysis of our spectral observations.

The aim of the first step of our modeling procedure was to get a fit of the synthetic "clean" (without spots) curve with the parts of the observed light curve that are most unaffected by the presence of spots - the durations of the two eclipses and the depth of the primary minimum (it is symmetrical, i.e. undistorted by spots) varying the orbital inclination $i$ in the range $80^{\circ}-90^{\circ}$, the relative radius $r_{1}$ of the primary star in the range $0.2-0.4$ and the ratio $k$ $\left(r_{2}=k r_{1}\right)$ in the range $0.7-0.9$. We assumed on this stage preliminary values of star temperatures of the components of RT And $T_{1}=6100 \mathrm{~K}$ and $T_{2}=4900 \mathrm{~K}$ corresponding to their spectral types.

The best fit was obtained for the values: $i=82^{\circ} \pm 0.1^{\circ}$; $r_{1}=0.32 \pm 0.01$ and $r_{2}=0.27 \pm 0.01$. So, the value of $k=0.84$ obtained by photometry coincides with that determined by the measurement of the rotational broadenings of the profiles of $\mathrm{H} \alpha$ and FeI 6678 lines. The value of the orbital inclination obtained by us is in good agreement with that obtained by Pain-Gaposchkin (1946), Mancuso et al. (1979, 1981), Arevalo \& Lazaro (1995, solution a) as well as by Wang \& $\mathrm{Lu}$ (1993).

Dapergolas et al. (1991) noted that the values of the ratio of the star radii $k$ and orbital inclination $i$ obtained by fitting the light curves of RT And by different authors, are grouped around 0.88 and 0.72 and respectively $82^{\circ}$ and $88^{\circ}$. Our values are therefore close to the first combination.

The second step of the modeling procedure was to fit the rest parts of the light curve, especially, to reproduce its almost linear course outside the eclipses. For that purpose we followed the procedure of Budding \& Zeilik (1985) and built the distortion curve, i.e. the difference between the observed curve and synthetic "clean" curve. The distortion curve is a result of the photometric effect of the spot(s). The shape of this curve (Fig. 14) allowed us to estimate that two cool spots could reproduce it. Taking into account the relatively large light contributions of the spots (reaching to $3-4 \%$ of the total light, Fig. 14) it was reasonable to put the spots on the primary star (if they were on the secondary they should be huge in size 


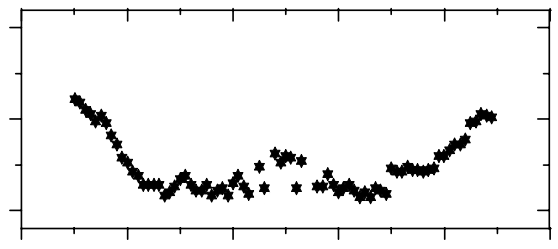

Fig. 14. Distortion light curve in $V$ band.

Table 1. Spot parameters determined by modeling of light curves during different seasons.

\begin{tabular}{ccccl}
\hline$\alpha$ & $\beta$ & $\lambda$ & $T$ & Reference \\
\hline 8.3 & 45 & 128 & 0 & Budding \& Zeilik (1987) \\
5.5 & 45 & 261 & 0 & Zeilik et al. (1988) \\
9.6 & 46 & 260 & 5000 & Zeilik et al. (1989a) \\
16.5 & 48 & 252 & 0 & Zeilik et al. (1989b) \\
13.3 & 63 & 257 & 5300 & Gordon et al. (1990) \\
15 & 70 & 110 & 5625 & Wand \& Lu (1993) \\
12 & 45 & 117 & 0 & Heckert (1995) \\
12 & 45 & 213 & 0 & Heckert et al. (1996) \\
12 & 44 & 238 & 0 & Heckert (1998) \\
21 & 50 & 112 & 4980 & this paper \\
21 & 45 & 237 & 4980 & \\
\hline
\end{tabular}

to produce the required light effect). We searched for a fit of the distortion curve varying the spot parameters: longitude $\lambda_{i}$; latitude $\beta_{i}$; angular size $\alpha_{i}$; temperature $T_{i}^{\mathrm{sp}}$ $(i=1,2)$. Different solutions were obtained for every color separately. However the aim was to find a fit in all colors with the same spot parameters.

We obtained the best fit with the following values: $\alpha_{1}=\alpha_{2}=21^{\circ} \pm 1^{\circ} ; \beta_{1}=50^{\circ} \pm 5^{\circ} ; \beta_{2}=45^{\circ} \pm 5^{\circ}$; $T_{1}^{\mathrm{sp}}=T_{2}^{\mathrm{sp}}=4980 \pm 60 \mathrm{~K} ; \lambda_{1}=112^{\circ} \pm 1^{\circ} ; \lambda_{2}=237^{\circ} \pm 1^{\circ}$. It turned out that the two spots are located symmetrically to the line connecting the star centers. The obtained angular sizes of the two spots mean that they cover $6.4 \%$ of the surface of the primary star. Their locations confirm the conclusion of Zeilik et al. (1989) that the spots of the short-period RS CVn stars appear at middle latitudes.

For comparison, Table 1 shows the spot parameters determined by modeling of light curves of RT And in different seasons. We suppose that the obtained smaller sizes of the spots partially due to the approximation $T^{\mathrm{sp}}=0 \mathrm{~K}$ used by the authors.

The last step of the modeling procedure was to fit the whole multicolor light curve varying star temperatures. So, we obtained the values $T_{1}=6150 \pm 10 \mathrm{~K}$ and $T_{2}=$ $4920 \pm 10 \mathrm{~K}$.

The final result of the complete fitting procedure is shown in Fig. 15. It is seen that the synthetic curve fits well the observed light curve. The small light depressions after the ends of the light minima are not reproduced by the synthetic curve. That means they are not due to the

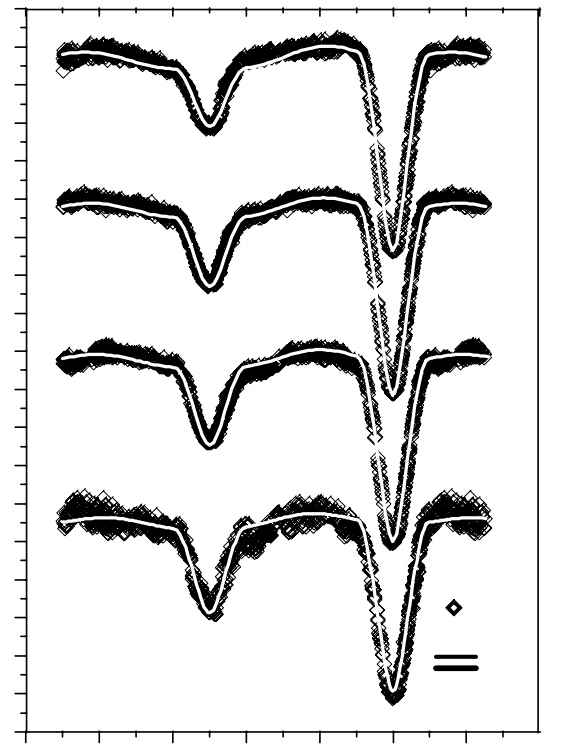

Fig. 15. The fitting light curves (blank lines) and observational light curves (blank diamonds). The curves in $V, R$ and $I$ bands are moved by 0.4 in intensity for a good visibility.

presence of spots but probably to extraphotospheric structures. The computer package cannot take into account their photometric effect.

The contribution of the primary star of RT And to the total light corresponding to the obtained system parameters is 0.83 in $B, 0.8$ in $V$ and 0.77 in $R$ band. These values are the same as those of Wang \& Lu (1993) while Budding \& Zeilik (1987), Zeilik et al. (1989b), Popper (1994) and Arevalo \& Lazaro (1995) obtain the slightly higher values of 0.86 in $V$ band.

The obtained ratio of the light contribution of the two stars of RT And in $R$ (the band of the two spectral lines investigated by us) $l=L_{2} / L_{1}=0.3$ almost coincides with the value obtained by measurement of their line fluxes in the spectral line FeI 6678, but is greater than that in $\mathrm{H} \alpha$.

The values of the absolute parameters of the star RT And determined by the different authors are quite different (Table 2). The reason probably is that they are obtained mostly by modeling of photometric data. But the light curve analyses are affected by the presence of spots which distribution and parameters changes from season to season due to the activity cycle. Criteria for separation of the effects of the spots from these of the eclipses and proximity are necessary for precise photometric modeling. Moreover, multicolor light curves on a standard photometric system are required in order to evaluate the star temperatures and luminosities. Most of the sets of photometric observations of RT And are not of this type. On the other hand high resolution spectral data are necessary for a precise determination of the masses of the star components. 
Table 2. Absolute parameters for RT And.

\begin{tabular}{llllllll}
\hline$i$ & $M_{1}$ & $M_{2}$ & $R_{1}$ & $R_{2}$ & $T_{1}$ & $T_{2}$ & Reference \\
\hline 89 & 1.04 & 0.68 & 1.15 & 0.83 & 6250 & 4800 & Budding \& Zeilik (1987) \\
86 & 1.05 & 0.68 & 1.17 & 0.84 & 6000 & 4900 & Zeilik et al. (1989) \\
87 & 1.24 & 0.91 & 1.27 & 0.91 & 6100 & 4900 & Arevalo \& Lazaro (1995) \\
88 & 1.24 & 0.91 & 1.26 & 0.90 & 6100 & 4730 & Popper (1994) \\
82 & 1.23 & 0.91 & 1.26 & 1.06 & 6150 & 4920 & this paper \\
\hline
\end{tabular}

\section{Discussion}

Precise values of star parameters of short-period RS CVn stars are necessary for checking the classical $\alpha \omega$ dynamo mechanism. According to it the surface activity in latetype stars is generated by both the differential rotation and convective motions of the star. The $\omega$ component, attributed to the differential rotation (a consequence of the Coriolis influence on the convective cells), drags the poloidal field lines into a stronger toroidal field. The $\alpha$ component, arising from the motions of the convective cells, twists these toroidal field lines and causes them to break through the surface, forming localized active regions detectable by their enhanced chromospheric emission. The dynamo number as a measure of the magnetic dynamo, is roughly inversely proportional to the square of the Rossby number $R_{0}=P_{\text {rot }} / \tau_{\mathrm{c}}\left(\tau_{\mathrm{c}}=l / V\right.$ where $l$ is the mixing length and $V$ is the convection velocity). Thus smaller values of the Rossby number correspond to a larger dynamo generation and presumably to larger magnetic activity.

Noyes et al. (1984) have obtained the following dependence of the turnover time at the base of the convection zone for dwarf star as a function of their $B-V$ :

$\log \left(\tau_{\mathrm{c}}\right)=1.362-0.166 x+0.025 x^{2}-5.323 x^{3}$

if $x>0$ where $x=1-(B-V)$ and

$\log \left(\tau_{\mathrm{c}}\right)=1.362-0.14 x$

if $x<0$.

Adopting the $B-V$ values for the primary and secondary star of RT And corresponding to their temperatures we obtained $\tau_{\mathrm{c}}^{1}=5.7$ and $\tau_{\mathrm{c}}^{2}=22.8$. Hence, the secondary star should show stronger chromospheric activity than the primary one. Our spectral observations confirm this.

Montes et al. (1995) found that systems with $\mathrm{H} \alpha$ emission above the continuum are all cooler than $5000 \mathrm{~K}$. The temperature of the primary star of RT And is above this value and one should not expect emission excess from it. Our spectral observations show only weak filled-in $\mathrm{H} \alpha$ profile of the primary star just before and after its eclipse and probably it is not connected with the primary itself.

The corresponding Rossby numbers of the two star components of RT And are $R_{0}^{1}=9533$ and $R_{0}^{2}=2437$ (their ratio is 3.9 ). So, our data confirm the relation derived from the dynamo theory between the activity level and the Rossby number, that means for the short-period RS CVn the stronger emission from their secondary stars.
Following the relation between the excess $\mathrm{H} \alpha$ emission surface flux and the Rossby number (Montes et al. 1995):

$F_{\mathrm{S}}(\mathrm{H} \alpha)=R_{0}^{-0.77}$

we calculated that the value of the ratio of the $\mathrm{H} \alpha$ fluxes from the primary and secondary star of RT And $F_{\mathrm{S}}^{1}(\mathrm{H} \alpha) / F_{\mathrm{S}}^{2}(\mathrm{H} \alpha)$ should be 0.35 . According to our observational data, this ratio is changeable during the orbital cycle and at most phases it is 0 because there is not detectable emission from the primary star.

In principle, the term "activity" refers to two kinds of phenomena. One is a type of surface variability caused by structures due to the magnetic field. On the other hand, activity also means the strengthening of emissions which show the existence of enhanced temperatures in "active regions" above those of "quiet regions". A knowledge of filling factors, i.e. the area of active regions on unresolved stellar disks, is a first step towards discovering the characteristic scales of activity. If the active regions are distributed fairly uniformly and close enough to each other, the filling factor will be close to 1 and the rotational modulation will be absent. Probably the secondary star of RT And is such a case.

Fernandez-Figueroa et al. (1994) found three different types of $\mathrm{H} \alpha$ profiles in chromospheric active binary systems: strong emission line, weak emission line with strong superposed absorption profile and filled-in absorption line profile.

The $\mathrm{H} \alpha$ emission from a chromospherically active binary with a light variation due to spots is expected to correlate with the light wave if the solar analogy works. Weiler (1978) found a marginal correlation between the $\mathrm{H} \alpha$ emission and the photometric wave in the RS CVn stars UX Ari and Z Her, in the sense that the strongest emission occurs at light minimum. Such correlations so far have been established for the stars II Peg (Vogt 1981), $\sigma$ Gem (Eker 1986), HR7275 (Eker 1989) and DH Leo (Newmark et al. 1990). A filling-in of the central core of the $\mathrm{H} \alpha$ line in chromospherically active binaries has been reported by Strassmeier et al. (1990), FernandezFigueroa et al. (1994), Eker et al. (1994). A different behavior of the $\mathrm{H}_{\alpha}$ profiles with the photometric wave has been noted and several assumptions about the emitting regions have been proposed: emission arising from regions concentrated around starspots (Vogt 1981; Newmark et al. 1990), from network-like structures (Huenemoerder et al. 1990), or from prominence-like material (Hall \& Ramsey $1992,1994)$. Whether one or the other of these sources dominates the emission excesses seems to be related to the luminosity class of the stars, i.e. with their surface gravity (Arevalo \& Lazaro 1999). It is accepted that the activity observed in the short-period RS CVn systems can be better explained by solar-type plages while in the longer period RS CVn stars plage-like structures do not appear to be the dominant source of the Balmer line excess emission.

The $\mathrm{H} \alpha$ profile, observed by us, of the primary component of RT And is symmetric out of the eclipses. There are not any features on it that might be connected with 
surface inhomogeneities. The primary star does not show $\mathrm{H} \alpha$ emission. However its FeI 6678 profile is not symmetric and there are emission features superposed on it at some phases. But we did not establish any regular phase behavior of these features.

In contrast, the $\mathrm{H} \alpha$ profiles and much more the FeI 6678 profiles of the secondary star of RT And are asymmetric and distorted by emission features at the most orbital phases. However the observed profiles are too weak for an investigation of their phase behavior. It seems that the secondary star of RT And shows both an enhanced chromospheric emission as a whole and features connected with active regions, but it is difficult to detect their photometric effect due to the small light contribution of the secondary star.

A large number of simultaneous observations in the lines that are activity indicators at different orbital phases are necessary for a better understanding of the star activity and to determine the individual contributions from plage regions, flaring, prominences, and the quiet chromosphere to the total profile. Moreover, the observational data should satisfy very strong requirements in order to allow to derive a correlation between the different appearances of the star activity of the short-period RS CVn stars.

We made the following considerations about the phase relation between the photometric and spectral behavior of stars with a surface activity. The cool spots should cause moving spectral features detectable as bumps on the spectral lines which phase should correlate with the spot visibility. If the emission from the chromosphere above the spots is enhanced then the emission features should be strongest at the phases when the line of sight is perpendicular to the star radius at the spot location. Then the observer sees the atmosphere above the spots unprojected on the star photosphere. If so, the phase of the maximum spot visibility $\phi^{\mathrm{sp}}$ and the phase of the strongest emission feature $\phi^{\mathrm{em}}$ should be related by the equation

$\phi^{\mathrm{em}}=\phi^{\mathrm{sp}} \pm 0.25$.

It is unreasonably to search for such or other correlation in our data due to the long time interval between our photometric and spectral observations (more than year) as well as due to the relative low $S / N$ of our spectral data.

\section{Conclusion}

The results from the analysis of our spectral and photometric data could be summarized as follows:

1. The lines $\mathrm{H} \alpha$ and FeI 6678 of RT And have double profiles that allow us to determine the masses of its star components and their rotational velocities.

2. The chromospheric activity of RT And is associated mostly with its secondary component.

3. The relative contribution of the secondary star is stronger in the FeI 6678 line than in $\mathrm{H} \alpha$.
4. There is a strong emission feature between the two profiles of FeI 6678 at phase 0.73 that could due to a transient extended emitting structure, probably flare.

5. Two cool spots on the primary star of RT And are responsible for the distortion of its light curve in 1998.

6. Both the measured rotational broadenings of the line profiles and the light curve analysis lead to the conclusion that the secondary star is oversized for its mass.

7. Due to long time interval between our spectral and photometric observations of RT And it is not reasonable to search for a relation of the photospheric spots and their effects on the spectral lines. Moreover, the relative low ratio $S / N$ of our spectra does not allow to detect spectral features from structures with a such small light contribution. Therefore we tried to establish only relations between the global parameters of the two star components of RT And.

8. We do not exclude the possibility the line $6678 \AA$ at least partially to due to HeI. This line is very sensitive to the presence of $\mathrm{He}$ and could be a result of a higher He abundance at an advanced evolutional state. The fact that the line $6678 \AA$ is relatively stronger from the secondary component of RT And than from the primary one as well as the fact that the secondary star is oversized for its mass imply such an interpretation. If the secondary star of RT And is subgiant then its surface layers should be He abundant.

Acknowledgements. The stay of the first two authors in Poland was supported by the Cracow Pedagogical University and by grants from Queen Jadwiga Foundation of the Jagiellonian Unversity. The authors thank to Prof. D. Gray and Dr. I. Iliev for the discussion about the identification of the spectral line $6678 \AA$.

\section{References}

Arevalo, M., \& Lazaro, C. 1995, AJ, 110, 1376

Arevalo, M., \& Lazaro, C. 1999, AJ, 118, 1015

Borkowski, J. 1988, Internal report of Torun (in Poland)

Bradstreet, D. 1993, Light Curve Modeling of Eclipsing Binary Stars, ed. E. Milone (Springer-Verlag New York), 151

Budding, E., \& Zeilik, M. 1987, ApJ, 319, 827

Budding, E., Cadouri, T., \& Gimenez, A. 1982, Ap\&SS, 88, 453

Dapergolas, A., Kontizas, E., \& Kontizas, M. 1991, IBVS, No. 3661

Dapergolas, A., Kontizas, E., \& Kontizas, M. 1994, IBVS, No. 4036

Dean, C. 1974, PASP, 86, 912

Eker, Z. 1986, MNRAS, 221, 947

Eker, Z. 1989, MNRAS, 238, 675

Eker, Z., Hall, D., \& Anderson, M. 1995, ApJS, 96, 581

Fernandez-Figueroa, M., Montes, D., De Castro, E., \& Cornide, M. 1994, ApJS, 90, 433 
Gordon, K. 1955, AJ, 53, 198

Gordon, S., Hall, D., Ledlow, M., Mann, E., \& Zeilik, M. 1990, IBVS, No. 3469

Gray, D. 1992, in The observations and analysis of stellar photospheres, Cambridge Astrophys. Ser., 20, 248

Hall, D. 1976, in Multiple Periodic Variable Stars, ed. W. Fitch (Dordrecht: Reidel), 287

Hall, J., \& Ramsey, L. 1992, AJ, 104, 1942

Hall, J., \& Ramsey, L. 1994, AJ, 107, 1149

Heckert, P. 1995, IBVS, No. 4224

Heckert, P., Beaver, M., \& Phillips, K. 1996, IBVS, No. 4384

Heckert, P. 1998, IBVS, No. 4656

Hempelmann, A., Hatzes, A., Kurster, M., \& Patkos, L. 1997, A\&A 317,125

Huenemoerder, D., Ramsey, L., \& Buzasi, D. 1990, ApJ, 350, 763

Huisong, T., \& Xuefu, L. 1987, A\&A, 172, 74

Kjurkchieva, D., Marchev, D., \& Ogloza, W. 2000a, A\&A, 354, 909

Kjurkchieva, D., Marchev, D., \& Ogloza, W. 2000b, Acta Astron., 50, 517

Krzesinski, J., \& Wojcik, K. 1993, A\&A, 280, 338

Kwee, K., \& van Woerden, H. 1956, Bull. Astron. Inst. Neth., 12,327

Mancuso, S., Milano, L., Russo, G., \& Sollazzo, C. 1979, Ap\&SS, 66, 475

Mancuso, S., Milano, L., Vittore, A., \& Budding 1981, in Photometric and Spectroscopic Binary Systems, ed. E. Carling, \& Z. Kopal (Dordrecht, Reidel), 313
Milano, L. 1981, in Photometric and Spectroscopic Binary Systems, ed. E. Carling, \& Z. Kopal (Dordrecht: Reidel), 331

Milano, L., Mancuso, S., Vittore, A., D’Osri, A., \& Marcozzi, S. 1986, Ap\&SS, 124, 83

Montes, D., Fernandez-Figueroa, M., De Castro, E., \& Cornide, M. 1995, A\&A, 294, 165

Newmark, J., Buzasi, D., Huenemoerder, D., et al. 1990, AJ, 100,560

Noyes, R., Weiss, N., \& Vaughan, A. 1984, ApJ, 287, 769

Pain-Gaposchkin, C. 1946, ApJ, 103, 191

Popper, D. 1994, AJ, 108, 1091

Smirnov, O., et al. 1992, Astron. Data Analysis Software and Systems I, ed. D. Worrall, et al., ASP Conf. Ser., 26, 344

Strassmeier, K., \& Fekel, F. 1990, A\&A, 230, 389

Vogt, S. 1981, ApJ, 247, 975

Wang, X., \& Lu, W. 1993, New Frontiers in Binary Star Research, ed. J. Leung, \& J. Nhu, ASP Conf. Ser., 38

Wilson, R. E. 1979, ApJ, 234, 1054

Wilson, R. E. 1983, DCMP and LCMP user's manual

Weiler, E. 1978, MNRAS, 182, 77

Zeilik, M., Elston, R., Henson, G., Schmolke, P., \& Smith, P. 1982, IBVS, No. 2090

Zeilik, M., Beckert, D., De Blasi, M., Ledlow, M., Rhodes, M., \& Williams, T. 1988, IBVS, No. 3173

Zeilik, M., Beckert, D., Cox, D., Dolsy, J., Ledlow, M., Newberry, M., \& Rhodes, M . 1989a, IBVS, No. 3301

Zeilik, M., Cox, D., De Blasi, C., \& Rhodes, M. 1989b, ApJ, 345,991 